\title{
Nrf2/ARE Pathway Involved in Oxidative Stress Induced by Paraquat in Human Neural Progenitor Cells
}

\author{
Tingting Dou, Mengling Yan, Xinjin Wang, Wen Lu, Lina Zhao, Dan Lou, \\ Chunhua Wu, Xiuli Chang, and Zhijun Zhou
}

School of Public Health and Key Laboratory of Public Health Safety of the Ministry of Education, Fudan University, Shanghai 200032, China

Correspondence should be addressed to Xiuli Chang; xlchang@fudan.edu.cn and Zhijun Zhou; zjzhou@shmu.edu.cn

Received 11 June 2015; Accepted 8 July 2015

Academic Editor: Michela Battistelli

Copyright (C) 2016 Tingting Dou et al. This is an open access article distributed under the Creative Commons Attribution License, which permits unrestricted use, distribution, and reproduction in any medium, provided the original work is properly cited.

Compelling evidences have shown that diverse environmental insults arising during early life can either directly lead to a reduction in the number of dopaminergic neurons or cause an increased susceptibility to neurons degeneration with subsequent environmental insults or with aging alone. Oxidative stress is considered the main effect of neurotoxins exposure. In this study, we investigated the oxidative stress effect of Paraquat (PQ) on immortalized human embryonic neural progenitor cells by treating them with various concentrations of PQ. We show that PQ can decrease the activity of SOD and CAT but increase MDA and LDH level. Furthermore, the activities of Cyc and caspase- 9 were found increased significantly at $10 \mu \mathrm{M}$ of PQ treatment. The cytoplasmic Nrf2 protein expressions were upregulated at $10 \mu \mathrm{M}$ but fell back at $100 \mu \mathrm{M}$. The nuclear Nrf2 protein expressions were upregulated as well as the downstream mRNA expressions of HO-1 and NQO1 in a dose-dependent manner. In addition, the proteins expression of PKC and CKII was also increased significantly even at $1 \mu \mathrm{M}$. The results suggested that Nrf2/ARE pathway is involved in mild to moderate PQ-induced oxidative stress which is evident from dampened Nrf2 activity and low expression of antioxidant genes in PQ induced oxidative damage.

\section{Introduction}

Agricultural chemicals are becoming increasingly potent environmental threats. Paraquat (PQ; $1,1^{\prime}$-dimethyl-4,4'-bipyridium) is a widely used fast-acting and nonselective contact herbicide, which is mainly accumulated in the lung, resulting in widespread reports for its pulmonary toxicity [1]. PQ has also been shown to cross the blood-brain barrier and enter the brain through a neutral amino acid carrier due to its structural homology to amino acids $[2,3]$. Animal experiments demonstrate that prolonged PQ exposure can result in its accumulation in different brain regions [4]. Extensive evidence demonstrates that PQ is linked to nigrostriatal damage and the emergence of Parkinson symptoms in epidemiological investigations and animal studies [5]. Importantly, compelling evidence from animal models has shown that diverse environmental factors arising during early life may either directly lead to a reduction in the number of dopamine neurons in substantia nigra or cause an increased susceptibility to degeneration of these neurons with subsequent environmental insults or with aging alone $[6,7]$. Moreover, some direct evidence shows that PQ has the ability to cross the placenta and it was found in higher concentrations in the placenta than in the mother's blood [8]. Exposure to PQ in the early life can produce progressive, permanent, and cumulative neurotoxicity of the nigrostriatal dopamine system and enhance vulnerability to subsequent environmental insults $[6,7]$. Our previous study in vitro suggested that $\mathrm{PQ}$ could reduce viability of human embryonic neural progenitor cells (hNPCs) by inducing oxidative stress and apoptosis [9]. Taken together, the persistence developmental neurotoxicity of $P Q$ which may contribute to later-inlife adverse effects needs more attention.

Although the mechanism of PQ neurotoxicity has not been completely clear, the importance of oxidative stress in it has been generally proved [10-12]. PQ can induce oxidative damage to various neurocytes like neurons and hippocampus cells [13-15]. PQ induces oxidative stress through producing 
reactive oxygen species (ROS), including hydrogen peroxide, superoxide anion, and hydroxyl radicals, and consuming nicotinamide adenine dinucleotide phosphate (NADPH), an important intracellular reducing agent to exert its toxicity. In addition, ROS is an important second messenger involved in activation or regulation of cell apoptosis. In the apoptosis pathway, caspase-dependent mitochondria pathway plays a decisive role. Divergent cellular stresses promote the release of caspase-activating factors, notably cytochrome c (Суc) from mitochondrial intermembrane space, to cytoplasm to combine with apoptotic protease activating factor-1 (Apaf1) and adenosine triphosphate (ATP) to form apoptosome assembly, activating caspase- 9 to further activate caspase3 , driving caspase cascade in cytoplasm, causing substrate proteolysis and cellular collapse to induce cell apoptosis [16, 17].

Therefore, the identification of the potential antioxidant pathway against oxidative damage had attracted intense interest. Among the multiple mechanisms, Nrf2-Keap1/ARE signal pathway is the most important endogenous antioxidant pathway discovered to date. While being attacked by ROS or other exogenous toxicant, the cytoplasm nuclear factor erythroid 2-related factor 2 (Nrf2) which was activated by dissociation with kelch-like ECH-associated protein (Keap1) entered the nucleus, binding to antioxidant reactive element (ARE), starting the transcription of corresponding downstream antioxidant molecules including phase II detoxifying enzymes [18] and antioxidative proteins [19, 20], to suppress the oxidative stress and maintain the redox balance. Several antioxidative enzymes and detoxifying enzymes levels had been downregulated in Nrf2 knockout mouse, which made the mouse more susceptive to toxin damage [21].

In addition, the developing brain is much more susceptible to being injured than the adult's brain $[22,23]$. Neural stem cells' self-renewal and multipotent differentiation capacity makes it an ideal model in studying neurodevelopmental toxicological mechanism $[24,25]$. In this study, we investigate the effects caused by PQ on the imbalance of oxidation and antioxidation and the role of the antioxidation pathway-the Nrf2/ARE pathway in PQ induced neurotoxicity in hNPCs.

\section{Material and Methods}

2.1. Chemicals and Solution. PQ was purchased from Sigma Chemical Co. (Sigma-Aldrich, Milan, Italy). ReNcell NSC Maintenance Medium and accutase were obtained commercially from Millipore (Temecula, CA). Epidermal growth factor (EGF) and basic fibroblast growth factor (FGF-2) were purchased from PeproTech. Laminin was purchased from Invitrogen (Carlsbad, CA, USA). Catalase Assay Kit, Malondialdehyde Assay Kit, Lactate Dehydrogenase Assay Kit, BCA Protein Assay Kit, Cell Lysis Buffer for Western and IP, goat anti-rabbit IgG-HRP, and goat anti-mouse IgG-HRP were obtained from Beyotime (Jiangsu, China). Total Superoxide Dismutase Assay Kit was purchased from Dojindo Molecular Technologies (Kumamoto, Japan). Tripure was obtained from Roche (Basel, Switzerland). The AMV first strand cDNA Synthesis Kit was purchased from MBI (Fermentas, Canada).
Real-time PCR Kit was obtained from Tiangen Biotech (Beijing, China). Rabbit anti-Nrf2 polyclonal antibody, rabbit anti-Keap1 polyclonal antibody, rabbit anti-PKC polyclonal antibody, and rabbit anti-CKII polyclonal antibody were purchased from GeneTex (San Antonio, USA). Mouse anti$\beta$-tubulin polyclonal antibody was purchased from Boster (Wuhan, China).

2.2. Cell Culture and PQ Treatment. Human neural progenitor cells (hNPCs) were obtained from Millipore (Temecula, CA). Frozen cells were thawed and expanded on laminincoated $100 \mathrm{~mm}$ diameter dish (Corning, Inc., Corning, NY) in complete medium containing fresh EGF $(20 \mathrm{ng} / \mathrm{mL})$ and FGF-2 $(20 \mathrm{ng} / \mathrm{mL})$. Cells were passaged when they were $80 \%$ confluent. After accutase dissociation and centrifugation at $300 \mathrm{~g}$ for $3 \mathrm{~min}$, cells were resuspended in complete medium and plated in laminin-coated dish (Corning, Inc., Corning, $\mathrm{NY}$ ). The cultures were incubated at $37^{\circ} \mathrm{C}$ in $5 \% \mathrm{CO}_{2}$. The medium was replaced every $24 \mathrm{~h}$.

The cells were plated at a density of $1 \times 10^{5} / \mathrm{mL}$ in laminincoated plates. When the cells are approximately 70\%-80\% confluent, PQ dissolved in PBS was added at concentrations ranging from 0,1 , and 10 to $100 \mu \mathrm{M}$ and the cultures were maintained for $24 \mathrm{~h}$.

2.3. Measurement of Biomarkers of Oxidative Stress. After treatment of PQ for $24 \mathrm{~h}$, the levels of methane dicarboxylic aldehyde (MDA) were determined as an indicator of lipid peroxidation. And the activities of superoxide dismutase (SOD), catalase (CAT), and MDA were measured using qualified kits $[26,27]$. The absorbance was obtained using a Microplate Reader (Biotek, USA) reading at corresponding wavelength.

2.4. Lactate Dehydrogenase (LDH) Leakage Assay. Oxidative stress-induced cytotoxicity was determined in a colorimetric assay based on the measurement of $\mathrm{LDH}$ released into the supernatant, which was determined using an LDH cytotoxicity assay kit according to the manufacturer's instructions [28]. The absorbance was obtained using a Microplate Reader (Biotek, USA) reading at $450 \mathrm{~nm}$.

2.5. RNA Purification, Reverse Transcription, and Quantitative Real-Time PCR. Cell samples were collected after being exposed to PQ for $24 \mathrm{~h}$. Total RNA was first extracted by Tripure following manufacturer instructions. The synthesis of the cDNA was performed utilizing an oligo(DT) primer and reverse transcriptase. All the primers were designed and synthesized based on Primer Premier Software 5.0 (PREMIER Biosoft International) by Sangon Biotech (Shanghai) Co., Ltd. The $\beta$-actin primers were $5^{\prime}$-CTCCATCCTGGCCTCGCTGT-3' (sense) and $5^{\prime}$-GCTGTCACCTTCACCGTTCC-3' (antisense; NM_001101). The HO-1 primers were $5^{\prime}$-TCGCCCCTGTCTACTTCC-3' (sense) and $5^{\prime}$-GCAGCTCCTGCAACTCCT-3' (antisense; NM_002133). The NQO-1 primers were $5^{\prime}$-GCCTAGCACAAGTACCACTCTTGGTC- ${ }^{\prime}$ (sense) and $5^{\prime}$-CTGAGGCAGGAGAATTGCTGGAACC-3' (antisense; NM_001025434). $\beta$-actin was used 
as the housekeeping gene. The real-time PCR was done using $2 \mu \mathrm{L}$ of cDNA, $0.5 \mu \mathrm{L}$ of each primer, and $2 \mu \mathrm{L}$ SYBR Green PCR Master Mix in a $10 \mu \mathrm{L}$ reaction volume. PCR program was a $10 \mathrm{~min}$ activation step at $95^{\circ} \mathrm{C}$, followed by 40 cycles of $94^{\circ} \mathrm{C}$ for $15 \mathrm{~s}, 55^{\circ} \mathrm{C}$ for $30 \mathrm{~s}$, and finally $72^{\circ} \mathrm{C}$ for $1 \mathrm{~min}$. Every sample was done in triplicate. The $\Delta \Delta \mathrm{Ct}$ method was applied for RNA relative expression quantification [9].

2.6. Western Blot Analysis of Cytoplasmic Nrf2, Nuclear Nrf2, Protein Kinase C (PKC), Casein Kinase II (CKII), Cyc, and Caspase-9. After treatment with $0,1,10$, and $100 \mu \mathrm{mol} / \mathrm{L}$ PQ for $24 \mathrm{~h}$, the protein extraction and western blot process were operated according to the previous study [9]. The immunoreactive proteins were detected by enhanced chemiluminescence using hyperfilm and enhanced chemiluminescence reagent (GE Healthcare, Little Chalfont, Buckinghamshire, UK) according to the manufacturer's instructions. Band intensities were quantified by densitometer analysis system and expressed as IOD (integrated optical density). Target protein densitometry values were adjusted to $\beta$-tubulin intensity and normalized to expression from the control sample.

2.7. Statistical Analysis. Data were analyzed with the SPSS 17.0 statistic program and expressed as means \pm standard deviations (SD). All data were analyzed by one-way analysis of variance (one-way ANOVA), followed by LSD- $t$ test for variance homogeneity and Kruskal-Wallis $H$ test for variance heterogeneity. Data obtained at each chemical concentration were compared with the controls. $p$-value $<0.05$ was considered significant.

\section{Results}

3.1. The Oxidative Damages Induced by PQ in hNPCs. Previous reports from our laboratory indicated that PQ can significantly reduce cells viability to $69 \%$ at concentration of $100 \mu \mathrm{M}$ while it had no effect at 0,1 , and $10 \mu \mathrm{M}$ [9]; we chose 0,1 and $10 \mu \mathrm{M}$ PQ which were no significant cytotoxic and $100 \mu \mathrm{M}$ PQ which was significant cytotoxic as the exposure concentration in this study. Considering the ROS generation induced by $\mathrm{PQ}$ at $10 \mu \mathrm{M}$ [9] and the balance between oxidation and antioxidation in normal circumstance, we first investigated the antioxidative molecules' SOD and CAT activity to research the oxidative mechanism after treatment with various concentrations of PQ. The two antioxidant enzyme activities both had a dose-dependent decrease with the significant decrease at $100 \mu \mathrm{M}$ PQ exposure compared to untreated group $(p<0.05)$ (Figure 1).

As the final metabolite of lipid peroxidation, MDA can change the construction and function of cell membrane, leading to the membrane breakage [29]. Besides, LDH which was released when the membrane damaged is considered a kind of sensitive markers of membrane breakage. Therefore, we detected the PQ induced oxidative damage via measuring the intracellular MDA and $\mathrm{LDH}$ activity. After treatment with various concentrations of $\mathrm{PQ}$, we found a significant dose-dependent increase of MDA and LDH activity with PQ concentrations as low as $10 \mu \mathrm{M}$ compared to untreated group $(p<0.05)$ (Figure 2), which were accordant with ROS [9].

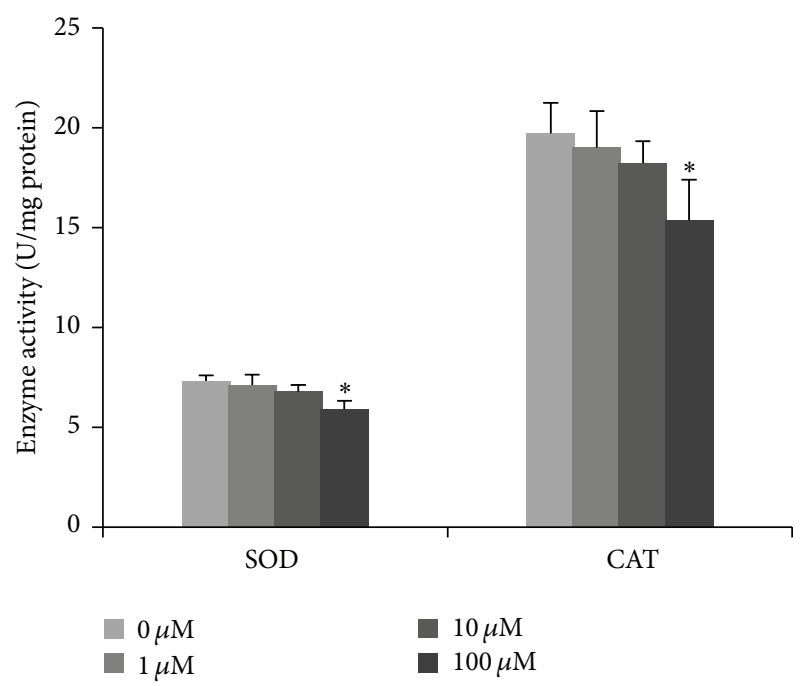

FIGURE 1: SOD and CAT activity in hNPCs upon exposure to different concentrations of PQ for $24 \mathrm{hr}$. Results are expressed as means \pm S.D. $(n=3)$. $*$ means $p<0.05$ when compared with the corresponding control group $(0 \mu \mathrm{M})$.

3.2. Nrf2/ARE Pathway Involved in Oxidative Stress Induced by $P Q$ in hNPCs. Nrf2-ARE-driven genes coordinately function to protect cells from $\mathrm{H}_{2} \mathrm{O}_{2}$-induced apoptosis in cell studies [30]. Toxic doses of $\mathrm{H}_{2} \mathrm{O}_{2}$ vary with the cell density, components in culture media, and the cell type studied. The following oxidative stress may result in apoptotic and/or necrotic cell death depending on a variety of factors. To examine the potential role of $\mathrm{Nrf} 2$ signaling pathway in preventing PQ induced oxidative stress in hNPCs, we detected the cytoplasmic and nuclear Nrf2 protein expression by western blot. As Figure 3 showed, the cytoplasmic Nrf2 expression was significantly upregulated to $181 \%$ at $10 \mu \mathrm{M}$ of PQ $(p<$ $0.05)$ but fell back to $116 \%$ at $100 \mu \mathrm{M}(p>0.05)$. The nuclear Nrf2 expression was significantly upregulated to $178 \%$ and $218 \%$ at 10 and $100 \mu \mathrm{M}(p<0.05)$.

As a result of nuclear Nrf2 increase, we examine Nrf2ARE-driven genes using real-time PCR after PQ treatment for $24 \mathrm{~h}$. HO-1 mRNA expression was slightly increased to $117 \%$ at $1 \mu \mathrm{M}$ and significantly increased to $175 \%$ and $221 \%$ at 10 and $100 \mu \mathrm{M}(p<0.05)$, respectively. NQO1 expression was slightly increased to $111 \%$ at $1 \mu \mathrm{M}$ and significantly increased to $215 \%$ and $220 \%$ at 10 and $100 \mu \mathrm{M}(p<0.01)$ (Figure 4$)$.

3.3. Activation of Protein Kinase (PKC and CKII) Induced by $P Q$ in hNPCs. Previous studies had discovered the involvement of protein kinase in phosphorylating Nrf2 and triggering its nuclear translocation in response to oxidative stress [31]. In our study, we used western blot to detect the intracellular PKC and CKII proteins expression after treatment with different concentrations of PQ for $24 \mathrm{~h}$ to observe the role of protein kinases in PQ induced Nrf2 activation. As Figure 5 showed, the intracellular PKC and CKII expressions were both significantly upregulated at even $1 \mu \mathrm{M}$ of PQ $(p<0.01)$. 


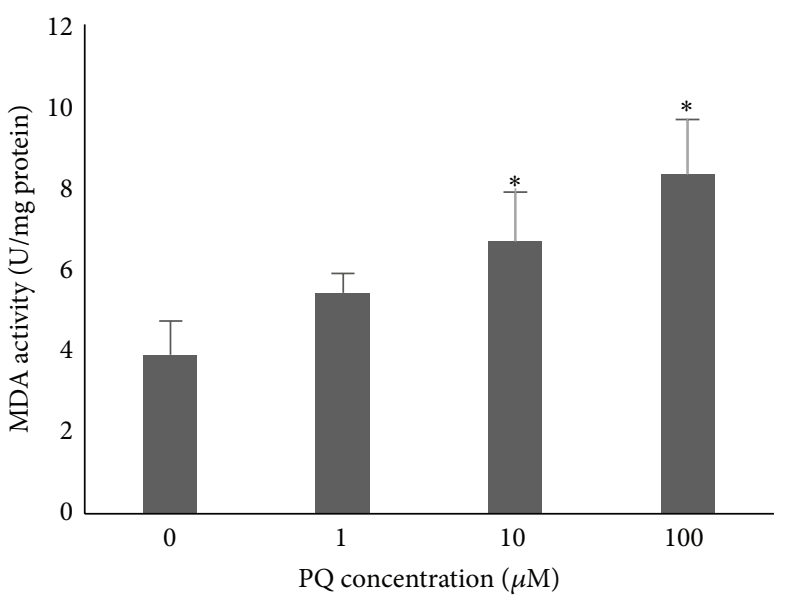

(a)

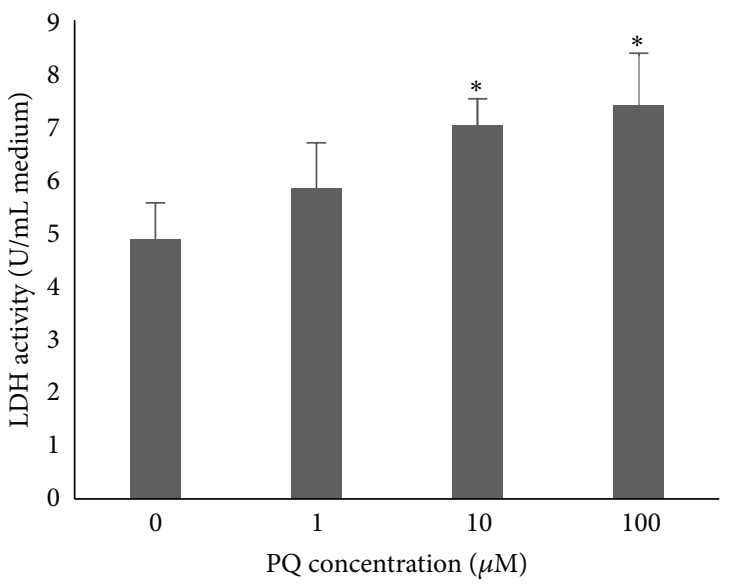

(b)

FIGURE 2: The oxidative damage of hNPCs induced by PQ treatment. (a) The intracellular MDA activity in hNPCs upon exposure to different concentrations of PQ. (b) The released LDH level of hNPCs upon exposure to different concentrations of PQ. Results are expressed as means \pm S.D. $(n=3)$. $*$ means $p<0.05$ when compared with the corresponding control group $(0 \mu \mathrm{M})$.

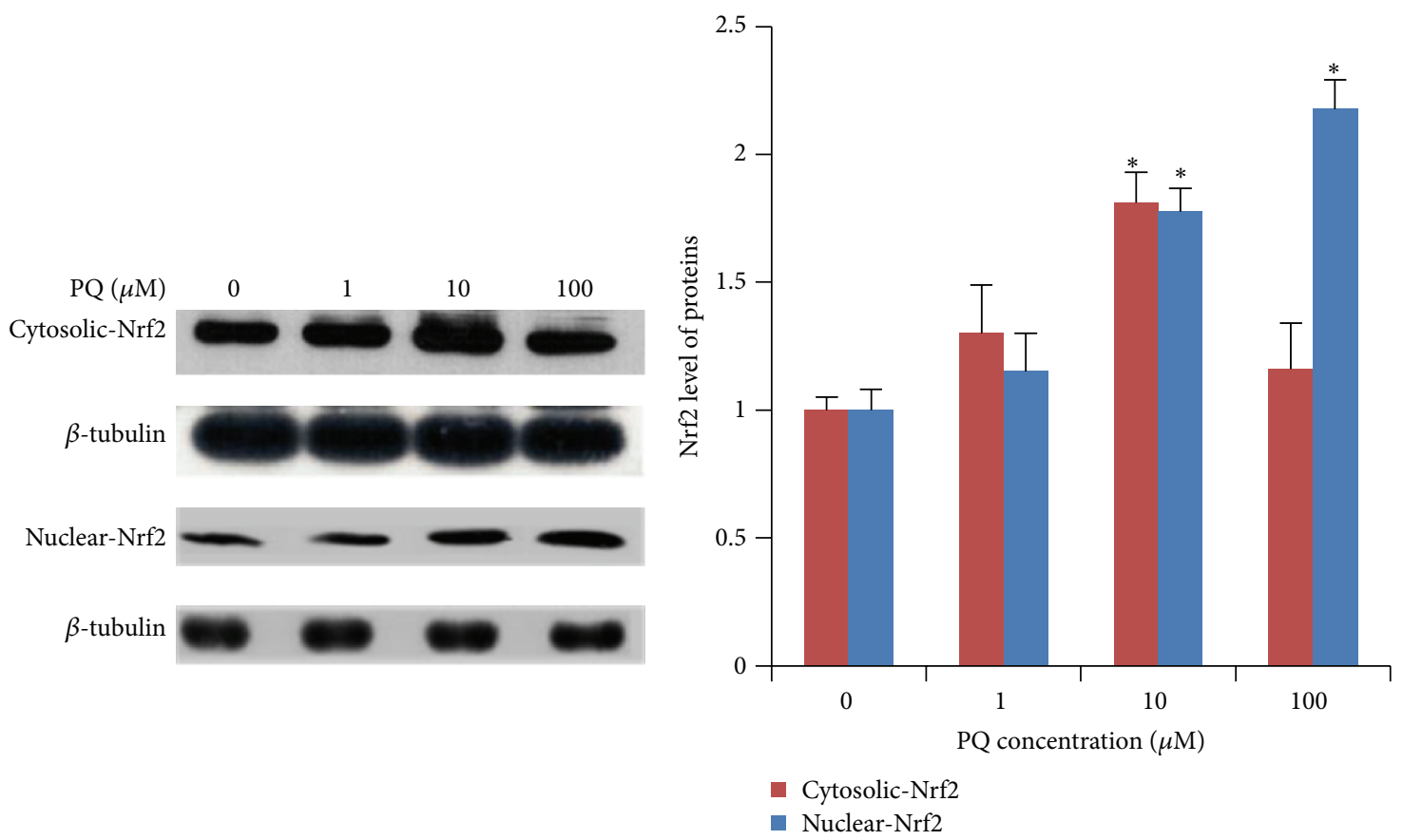

(a)

(b)

Figure 3: The expression of cytoplasmic and nuclear Nrf2 proteins in hNPCs after exposure to different concentrations of PQ. (a) Electrophoretic band of cytoplasmic and nuclear Nrf2 proteins by western blot. (b) Quantification of cytoplasmic and nuclear Nrf2 proteins expression. Results are expressed as means \pm S.D. $(n=3)$. $*$ and $* *$ mean $p<0.05$ and $p<0.01$ when compared with the corresponding control group $(0 \mu \mathrm{M})$.

3.4. Effect of PQ on Apoptotic Cell Death Signaling Pathways of $h N P C s$. Oxidative stress had been shown to activate caspase-dependent apoptotic cell death signaling pathways [32]. Cyc combined with Apaf-1 and subsequently activated caspase-9 to initiate downstream effector caspases to cause the cell apoptosis $[33,34]$. Herein, we examined the protein expressions of Cyc and caspase-9. Figure 6 showed that Cyc and caspase- 9 protein expressions were both significantly increased in $10 \mu \mathrm{M}$ PQ treatment group compared with the control $(p<0.05)$. 


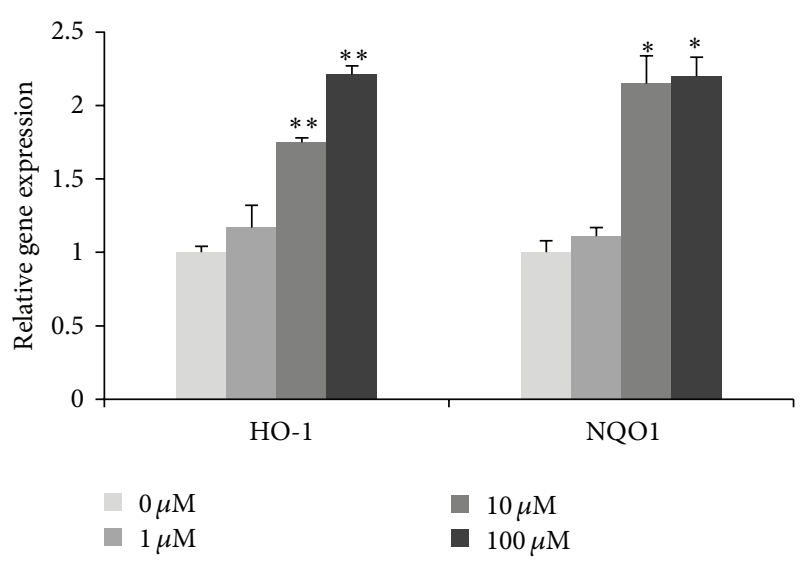

FIGURE 4: The gene expression of HO-1 and NQO1 in hNPCs after exposure to different concentrations of $\mathrm{PQ}$. Results are expressed as means \pm S.D. $(n=3) . *$ and $* *$ mean $p<0.05$ and $p<0.01$ when compared with the corresponding control group $(0 \mu \mathrm{M})$.

\section{Discussion}

In the present study, we demonstrate that PQ can directly produce toxicity to hNPCs by inducing ROS generation and decreasing SOD and CAT activity which resulted in redox imbalance and oxidative damage. In particular, we found that PQ induced oxidative stress can activate the Nrf2-Keap1/ARE signaling pathway to initiate the downstream antioxidant responsive elements including $\mathrm{HO}-1$ and NQO1 mRNAs expression to prevent the oxidative damage. Additionally, we observed that PQ can activate PKC and CKII which were involved in the phosphorylation of Nrf2, revealing that PKC and CKII may play an indirect part in antioxidative stress.

As one of the most widely used herbicides in the world, PQ can induce damage to various organs or cells [35-37]. To the nervous system, because of the structural similarity to the parkinsonism-inducing neurotoxic agent 1-methyl-4-phenyl1, 2, 3, 6-tetrahydropyridine (MPTP), PQ is considered to be a possible environmental risk factor for neurodegenerative disorders like Parkinson's disease (PD) $[38,39]$. In addition, the developing brain is much more susceptible to be injured than the adult's brain [21, 22]. Therefore, PQ developmental neurotoxicity deserves more attention. It was reported that PQ could inhibit the proliferation and disrupt the differentiation of neural precursor cells in vitro studies [40]. Also, in our previous study, we found the concentration of PQ reducing the hNPCs viability $(100 \mu \mathrm{M})$ [9] was lower than other cell types $[41,42]$, suggesting the sensitivity of hNPCs to PQ induced toxicity. Based on the effect of PQ on hNPCs viability, in this study, we chose $0,1,10$, and $100 \mu \mathrm{M}$ as the exposure concentrations.

Because of its extensive effects on cell proliferation, differentiation, apoptosis, and signal transduction, ROS plays an important role in neurotoxicity. Several studies have suggested that PQ could cause dopaminergic neurons and hippocampal neurons damage via generating ROS to cause oxidative damage to brain mitochondria $[15,43]$. Our previous study had also shown that PQ exposure could cause a dose-dependent increase in ROS production and significant increases were observed when PQ doses were increased to $10 \mu \mathrm{M}$ [9]. Besides, $\mathrm{LDH}$ which is released when the membrane damaged is considered a kind of sensitive markers of membrane breakage. Similarly, our study showed that PQ increased the LDH and MDA levels. These results suggest that PQ induced the production of lipid peroxidation and oxidative damage in hNCPs.

As a sensitive receptor for oxidative stress, Nrf2/Keap1 signaling pathway played a crucial role in preventing cells from apoptosis, stress, inflammation, and tumor [20]. It was the most important intrinsic antioxidative stress pathway yet discovered $[44,45]$. When the Nrf2/Keap1 signaling pathway was activated, Nrf2 was uncoupled from inhibitor proteinKeap1 and accessed to nucleus to bind with ARE, leading to the transcription of the downstream target antioxidative genes, and sequentially improved cell's antioxidative stress ability [46, 47]. Similarly, in our study, $10 \mu \mathrm{M}$ PQ induced oxidative stress can significantly activate the Nrf2 pathway to prevent the oxidative stress via increasing the level of both cytoplasmic and nuclear Nrf2 protein and the Nrf2 downstream antioxidative genes, HO-1 and NQO1, which meant the activation of Nrf2/Keap1 signaling pathway to increase nuclear antioxidative genes expression to prevent $10 \mu \mathrm{M}$ PQ caused oxidative stress.

In other way, normal cells can eliminate the redundant ROS through antioxidant enzyme including SOD and CAT to prevent the oxidative damage. But severe exposure to toxicant could increase the oxidation but decrease the antioxidation to damage the oxidative balance. Previous studies have showed that SOD and CAT in dopaminergic neurons were all decreased after PQ exposure $[41,48]$. Similarly, our study showed that higher level of PQ $(100 \mu \mathrm{M})$ can significantly inhibit antioxidant enzymes SOD and CAT activity, which meant the imbalance of oxidation and the severity of $100 \mu \mathrm{M}$ PQ induced oxidative damage. Enhanced nuclear Nrf2 expression was further augmented by PQ treatment to further activate downstream antioxidative genes $\mathrm{HO}-1$ and NQO1 expressions to defend the oxidative damage. The level of cytoplasmic Nrf2 fell back at $100 \mu \mathrm{M}$. Similarly, a previous study also has discovered that higher concentration of PQ exposure $(0.5 \mathrm{mmol} / \mathrm{L})$ could inhibit neuroblastoma Nrf2 protein level [49].

In addition, some protein kinases like PKC and CKII could also induce the Nrf2 protein phosphorylation to influence its activity $[31,50]$. In our study, the intracellular PKC and CKII protein levels were elevated with the increasing of PQ concentration even at $1 \mu \mathrm{M}$, which revealed the correlation between Nrf2 activation and its phosphorylation caused by PKC and CKII.

However, as an important second messenger involved in regulation of cell apoptosis, high level of ROS induced by PQ could inevitably cause mitochondria damage and cell apoptosis [51, 52]. In our study, the mitochondria released Cyc level increased at $10 \mu \mathrm{M}$, which resulted in the increase of caspase-9. That was consistent with the cells apoptosis and cells viability alteration discovered in previous study [9], suggesting the obvious cells damage caused by high concentration $(100 \mu \mathrm{M})$ of PQ. 


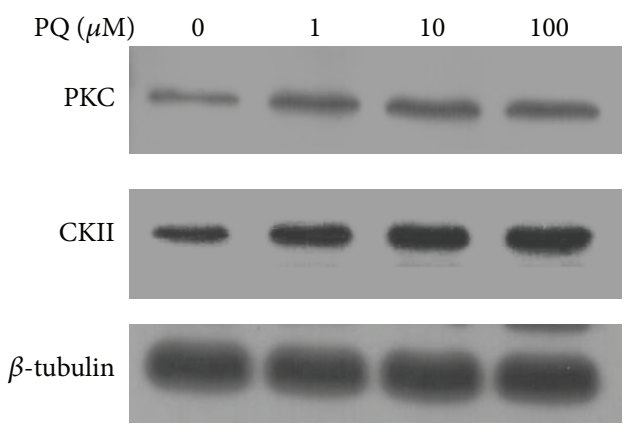

(a)

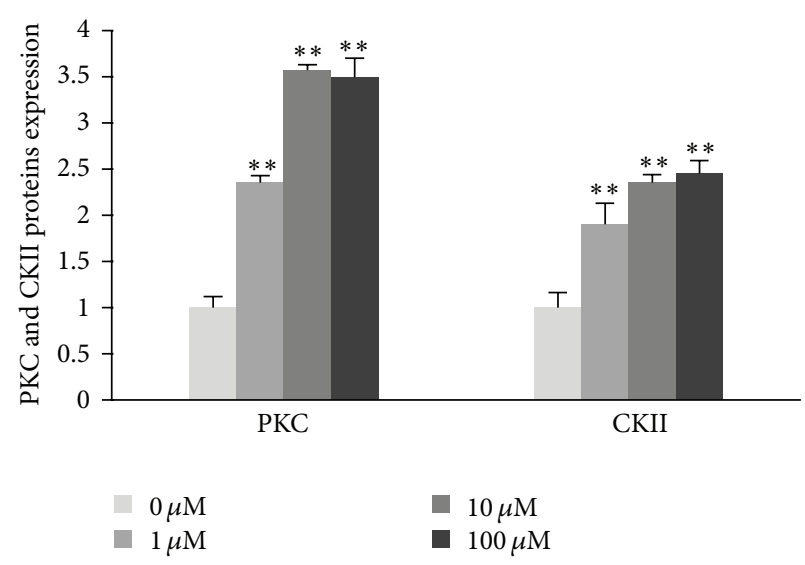

(b)

FIGURE 5: The expression of PKC and CKII proteins in hNPCs after exposure to different concentrations of PQ. (a) Electrophoretic band of PKC and CKII proteins by western blot. (b) Quantification of PKC and CKII proteins expression. Results are expressed as means \pm S.D. $(n=3) . * *$ means $p<0.01$ when compared with the corresponding control group $(0 \mu \mathrm{M})$.

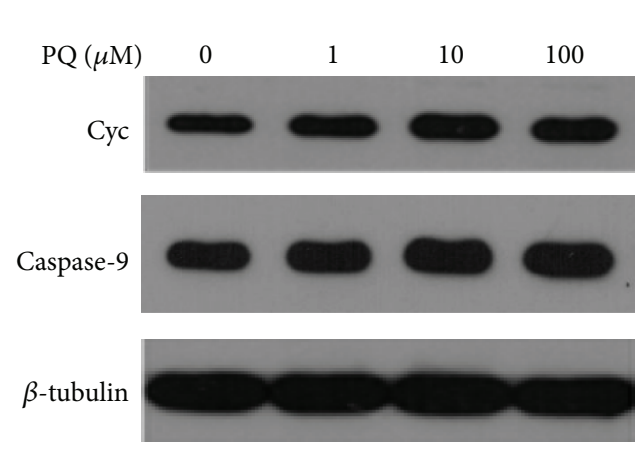

(a)

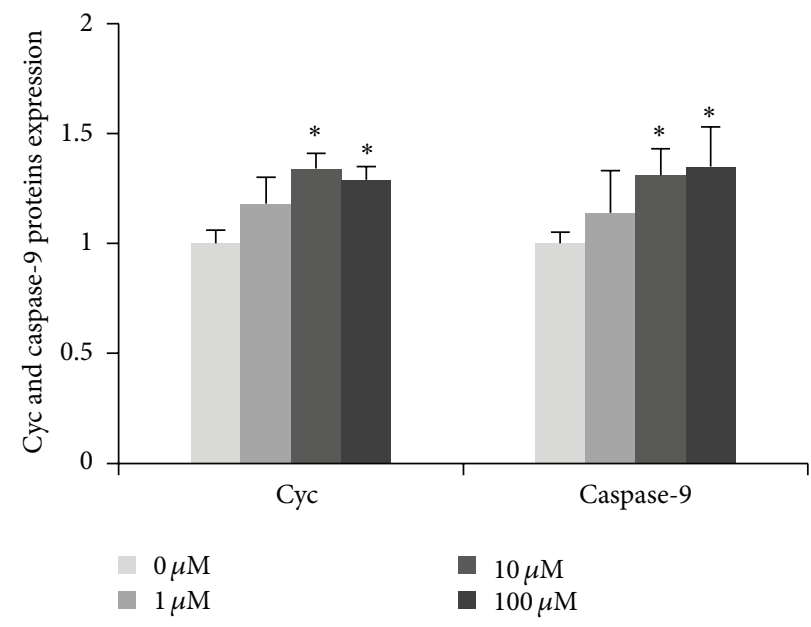

(b)

FIGURE 6: The expression of Cyc and caspase-9 proteins in hNPCs after exposure to different concentrations of PQ. (a) Electrophoretic band of Cyc and caspase-9 proteins by western blot. (b) Quantification of Cyc and caspase-9 proteins expression. Results are expressed as means \pm S.D $(n=3)$. * means $p<0.05$ when compared with the corresponding control group $(0 \mu \mathrm{M})$.

In general, $\mathrm{PQ}$ exposure could significantly induce oxidative stress and cause oxidative imbalance. Low concentration $(10 \mu \mathrm{M})$ of PQ caused moderate stress could activate Nrf2 pathway to prevent cells from $P Q$ induced oxidative damage; however, the Nrf2 pathway protection was ineffective in high concentration $(100 \mu \mathrm{M})$ and caused severe stress, which has finally resulted in decrease of cell ability and increase of cell apoptosis [9]. Considering the protection of Nrf2 pathway in PQ induced damage, it may be supposed as a potential and effective therapeutic target for oxidative damage related disease in clinical therapy.

\section{Conclusion}

We provide important evidence suggesting that $\mathrm{PQ}$ has direct toxicity to cause irreversible apoptosis to hNPCs which was associated with the elevated oxidative stress. In addition, PQ induced oxidative stress and redox imbalance could activate the Nrf2/ARE signaling pathway to prevent the oxidative stress via initiating the downstream antioxidant responsive element like HO-1 and NQO1 mRNAs expression. In particular, we also observed that PKC and CKII may be involved in the phosphorylation of Nrf2, revealing that PKC and CKII may play an indirect role in antioxidative stress. 


\section{Conflict of Interests}

The authors declare that there is no conflict of interests regarding the publication of this paper.

\section{Authors' Contribution}

Tingting Dou and Mengling Yan contributed equally to this work.

\section{Acknowledgments}

This research was supported by the National Natural Science Funds (NSFC 81472996, and 81072324, China) and Shanghai National Natural Science Funds (10ZR1401700). We thank Dr. Qiangen Wu for helpful comments on this paper.

\section{References}

[1] Y.-W. Chen, Y.-T. Yang, D.-Z. Hung, C.-C. Su, and K.-L. Chen, "Paraquat induces lung alveolar epithelial cell apoptosis via Nrf2-regulated mitochondrial dysfunction and ER stress," Archives of Toxicology, vol. 86, no. 10, pp. 1547-1558, 2012.

[2] R. J. Dinis-Oliveira, F. Remião, H. Carmo et al., "Paraquat exposure as an etiological factor of Parkinson's disease," NeuroToxicology, vol. 27, no. 6, pp. 1110-1122, 2006.

[3] C.-Y. Lee, C.-H. Lee, C.-C. Shih, and H.-H. Liou, "Paraquat inhibits postsynaptic AMPA receptors on dopaminergic neurons in the substantia nigra pars compacta," Biochemical Pharmacology, vol. 76, no. 9, pp. 1155-1164, 2008.

[4] K. Prasad, E. Tarasewicz, J. Mathew et al., "Toxicokinetics and toxicodynamics of paraquat accumulation in mouse brain," Experimental Neurology, vol. 215, no. 2, pp. 358-367, 2009.

[5] Registration Eligibility Decision (RED), Office of Prevention, Pesticides and Toxic Substances, United States Environmental Protection Agency, EPA 738-F-96-018: Paraquat dichloride, US EPA, Washington, DC, USA, 1997, http://www.epa.gov/pesticides/reregistration/status.htm.

[6] K. R. Reuhl, "Delayed expression of neurotoxicity: the problem of silent damage," NeuroToxicology, vol. 12, no. 3, pp. 341-346, 1991.

[7] B. K. Barlow, D. A. Cory-Slechta, E. K. Richfield, and M. Thiruchelvam, "The gestational environment and Parkinson's disease: evidence for neurodevelopmental origins of a neurodegenerative disorder," Reproductive Toxicology, vol. 23, no. 3, pp. 457470, 2007.

[8] A. M. Tsatsakis, K. Perakis, and E. Koumantakis, "Experience with acute paraquat poisoning in Crete," Veterinary and Human Toxicology, vol. 38, no. 2, pp. 113-117, 1996.

[9] X. Chang, W. Lu, T. Dou et al., "Paraquat inhibits cell viability via enhanced oxidative stress and apoptosis in human neural progenitor cells," Chemico-Biological Interactions, vol. 206, no. 2, pp. 248-255, 2013.

[10] J. S. Bus, S. D. Aust, and J. E. Gibson, "Superoxide- and singlet oxygen-catalyzed lipid peroxidation as a possible mechanism for paraquat (methyl viologen) toxicity," Biochemical and Biophysical Research Communications, vol. 58, no. 3, pp. 749-755, 1974.

[11] J. S. Bus, S. Z. Cagen, M. Olgaard, and J. E. Gibson, "A mechanism of paraquat toxicity in mice and rats," Toxicology and Applied Pharmacology, vol. 35, no. 3, pp. 501-513, 1976.
[12] C.-S. Lai and L. H. Piette, "Hydroxyl radical production involved in lipid peroxidation of rat liver microsomes," Biochemical and Biophysical Research Communications, vol. 78, no. 1, pp. 51-59, 1977.

[13] D. A. Drechsel and M. Patel, "Chapter 21 paraquat-induced production of reactive oxygen species in brain mitochondria," Methods in Enzymology, vol. 456, pp. 381-393, 2009.

[14] A. Zaidi, D. Fernandes, J. L. Bean, and M. L. Michaelis, "Effects of paraquat-induced oxidative stress on the neuronal plasma membrane $\mathrm{Ca}^{2+}$-ATPase," Free Radical Biology and Medicine, vol. 47, no. 10, pp. 1507-1514, 2009.

[15] Q. Chen, Y. Niu, R. Zhang et al., "The toxic influence of paraquat on hippocampus of mice: involvement of oxidative stress," NeuroToxicology, vol. 31, no. 3, pp. 310-316, 2010.

[16] C. Adrain and S. J. Martin, "The mitochondrial apoptosome: a killer unleashed by the cytochrome seas," Trends in Biochemical Sciences, vol. 26, no. 6, pp. 390-397, 2001.

[17] S. Sadasivam, S. Gupta, V. Radha, K. Batta, T. K. Kundu, and G. Swarup, "Caspase-1 activator Ipaf is a p53-inducible gene involved in apoptosis," Oncogene, vol. 24, no. 4, pp. 627-636, 2005.

[18] R. Munday and C. M. Munday, "Induction of phase II detoxification enzymes in rats by plant-derived isothiocyanates: comparison of allyl isothiocyanate with sulforaphane and related compounds," Journal of Agricultural and Food Chemistry, vol. 52, no. 7, pp. 1867-1871, 2004.

[19] J. A. Rubiolo, G. Mithieux, and F. V. Vega, "Resveratrol protects primary rat hepatocytes against oxidative stress damage: activation of the Nrf2 transcription factor and augmented activities of antioxidant enzymes," European Journal of Pharmacology, vol. 591, no. 1-3, pp. 66-72, 2008.

[20] S. K. Kim, J. W. Yang, M. R. Kim et al., "Increased expression of Nrf2/ARE-dependent anti-oxidant proteins in tamoxifenresistant breast cancer cells," Free Radical Biology and Medicine, vol. 45, no. 4, pp. 537-546, 2008.

[21] H.-Y. Cho, A. E. Jedlicka, S. P. M. Reddy et al., "Role of NRF2 in protection against hyperoxic lung injury in mice," The American Journal of Respiratory Cell and Molecular Biology, vol. 26, no. 2, pp. 175-182, 2002.

[22] L. Claudio, W. C. Kwa, A. L. Russell, and D. Wallinga, “Testing methods for developmental neurotoxicity of environmental chemicals," Toxicology and Applied Pharmacology, vol. 164, no. 1, pp. 1-14, 2000.

[23] H. A. Tilson, "Neurotoxicology risk assessment guidelines: developmental neurotoxicology," NeuroToxicology, vol. 21, no. 1-2, pp. 189-194, 2000.

[24] B. A. Reynolds, W. Tetzlaff, and S. Weiss, "A multipotent EGFresponsive striatal embryonic progenitor cell produces neurons and astrocytes," Journal of Neuroscience, vol. 12, no. 11, pp. 45654574, 1992.

[25] R. Donato, E. A. Miljan, S. J. Hines et al., "Differential development of neuronal physiological responsiveness in two human neural stem cell lines," BMC Neuroscience, vol. 8, article 36, 2007.

[26] J.-M. Zhang, H.-C. Wang, H.-X. Wang et al., "Oxidative stress and activities of caspase-8, -9 , and -3 are involved in cryopreservation-induced apoptosis in granulosa cells," European Journal of Obstetrics \& Gynecology and Reproductive Biology, vol. 166, no. 1, pp. 52-55, 2013.

[27] L. Min, S. He, Q. Chen, F. Peng, H. Peng, and M. Xie, "Comparative proteomic analysis of cellular response of human airway epithelial cells (A549) to benzo(a)pyrene," Toxicology Mechanisms and Methods, vol. 21, no. 5, pp. 374-382, 2011. 
[28] S. M. Hussain and J. M. Frazier, "Cellular toxicity of hydrazine in primary rat hepatocytes," Toxicological Sciences, vol. 69, no. 2, pp. 424-432, 2002.

[29] P. Karakashev, L. Petrov, and A. Alexandrova, "Paraquatinduced lipid peroxidation and injury in Ehrlich ascites tumor cells," Neoplasma, vol. 47, no. 2, pp. 122-124, 2000.

[30] J. Li, D. Johnson, M. Calkins, L. Wright, C. Svendsen, and J. Johnson, "Stabilization of Nrf2 by tBHQ confers protection against oxidative stress-induced cell death in human neural stem cells," Toxicological Sciences, vol. 83, no. 2, pp. 313-328, 2005.

[31] H.-C. Huang, T. Nguyen, and C. B. Pickett, "Phosphorylation of Nrf2 at Ser-40 by protein kinase $\mathrm{C}$ regulates antioxidant response element-mediated transcription," The Journal of Biological Chemistry, vol. 277, no. 45, pp. 42769-42774, 2002.

[32] B. Peter, M. Wartena, H. H. Kampinga, and A. W. T. Konings, "Role of lipid peroxidation and DNA damage in paraquat toxicity and the interaction of paraquat with ionizing radiation," Biochemical Pharmacology, vol. 43, no. 4, pp. 705-715, 1992.

[33] C.-C. Lin, C.-L. Kuo, M.-H. Lee et al., "Wogonin triggers apoptosis in human osteosarcoma U-2 OS cells through the endoplasmic reticulum stress, mitochondrial dysfunction and caspase-3-dependent signaling pathways," International Journal of Oncology, vol. 39, no. 1, pp. 217-224, 2011.

[34] Y. Liang and J. P. Sundberg, "SHARPIN regulates mitochondriadependent apoptosis in keratinocytes," Journal of Dermatological Science, vol. 63, no. 3, pp. 148-153, 2011.

[35] M. Tsukamoto, Y. Tampo, M. Sawada, and M. Yonaha, "Paraquat-induced oxidative stress and dysfunction of the glutathione redox cycle in pulmonary microvascular endothelial cells," Toxicology and Applied Pharmacology, vol. 178, no. 2, pp. 82-92, 2002.

[36] E. Weidauer, T. Lehmann, A. Rämisch, E. Röhrdanz, and H. Foth, "Response of rat alveolar type II cells and human lung tumor cells towards oxidative stress induced by hydrogen peroxide and paraquat," Toxicology Letters, vol. 151, no. 1, pp. 6978, 2004.

[37] J. Peng, F. F. Stevenson, M. L. Oo, and J. K. Andersen, "Ironenhanced paraquat-mediated dopaminergic cell death due to increased oxidative stress as a consequence of microglial activation," Free Radical Biology \& Medicine, vol. 46, no. 2, pp. 312$320,2009$.

[38] C. Hertzmann, M. Wiens, D. Bowering, B. Snow, and D. Calne, "Parkinson's disease: a case-control study of occupational and environmental risk factors," American Journal of Industrial Medicine, vol. 17, no. 3, pp. 349-355, 1990.

[39] K. M. Semchuk, E. J. Love, and R. G. Lee, "Parkinson's disease and exposure to agricultural work and pesticide chemicals," Neurology, vol. 42, no. 7, pp. 1328-1335, 1992.

[40] H. T. Hogberg, A. Kinsner-Ovaskainen, T. Hartung, S. Coecke, and A. K. Bal-Price, "Gene expression as a sensitive endpoint to evaluate cell differentiation and maturation of the developing central nervous system in primary cultures of rat cerebellar granule cells (CGCs) exposed to pesticides," Toxicology and Applied Pharmacology, vol. 235, no. 3, pp. 268-286, 2009.

[41] M. Takizawa, K. Komori, Y. Tampo, and M. Yonaha, "Paraquatinduced oxidative stress and dysfunction of cellular redox systems including antioxidative defense enzymes glutathione peroxidase and thioredoxin reductase," Toxicology in Vitro, vol. 21, no. 3, pp. 355-363, 2007.

[42] W. Yang and E. Tiffany-Castiglioni, “The bipyridyl herbicide paraquat produces oxidative stress-mediated toxicity in human neuroblastoma SH-SY5Y cells: relevance to the dopaminergic pathogenesis," Journal of Toxicology and Environmental Health A, vol. 68, no. 22, pp. 1939-1961, 2005.

[43] M. G. Purisai, A. L. McCormack, S. Cumine, J. Li, M. Z. Isla, and D. A. Di Monte, "Microglial activation as a priming event leading to paraquat-induced dopaminergic cell degeneration," Neurobiology of Disease, vol. 25, no. 2, pp. 392-400, 2007.

[44] G. Y. Wu, Y.-Z. Fang, S. Yang, J. R. Lupton, and N. D. Turner, "Glutathione metabolism and its implications for health," Journal of Nutrition, vol. 134, no. 3, pp. 489-492, 2004.

[45] T. Nguyen, P. J. Sherratt, and C. B. Pickett, "Regulatory mechanisms controlling gene expression mediated by the antioxidant response element," Annual Review of Pharmacology and Toxicology, vol. 43, pp. 233-260, 2003.

[46] M. McMahon, N. Thomas, K. Itoh, M. Yamamoto, and J. D. Hayes, "Dimerization of substrate adaptors can facilitate cullin-mediated ubiquitylation of proteins by a 'Tethering' mechanism-a two-site interaction model for the Nrf2-Keap1 complex," Journal of Biological Chemistry, vol. 281, no. 34, pp. 24756-24768, 2006.

[47] Y. Luo, A. L. Eggler, D. Liu, G. Liu, A. D. Mesecar, and R. B. van Breemen, "Sites of alkylation of human Keapl by natural Chemoprevention agents," Journal of the American Society for Mass Spectrometry, vol. 18, no. 12, pp. 2226-2232, 2007.

[48] J.-P. Ren, Y.-W. Zhao, and X.-J. Sun, “Toxic influence of chronic oral administration of paraquat on nigrostriatal dopaminergic neurons in C57BL/6 mice," Chinese Medical Journal, vol. 122, no. 19, pp. 2366-2371, 2009.

[49] W. Yang, E. Tiffany-Castiglioni, M.-Y. Lee, and I.-H. Son, "Paraquat induces cyclooxygenase-2 (COX-2) implicated toxicity in human neuroblastoma SH-SY5Y cells," Toxicology Letters, vol. 199, no. 3, pp. 239-246, 2010.

[50] P. L. Apopa, X. He, and Q. Ma, "Phosphorylation of Nrf2 in the transcription activation domain by casein kinase 2 (CK2) is critical for the nuclear translocation and transcription activation function of Nrf2 in IMR-32 neuroblastoma cells," Journal of Biochemical and Molecular Toxicology, vol. 22, no. 1, pp. 63-76, 2008.

[51] C.-L. Huang, Y.-C. Lee, Y.-C. Yang, T.-Y. Kuo, and N.-K. Huang, "Minocycline prevents paraquat-induced cell death through attenuating endoplasmic reticulum stress and mitochondrial dysfunction," Toxicology Letters, vol. 209, no. 3, pp. 203-210, 2012.

[52] Y.-C. Wang, C.-M. Lee, L.-C. Lee et al., "Mitochondrial dysfunction and oxidative stress contribute to the pathogenesis of spinocerebellar ataxia type 12 (SCA12)," The Journal of Biological Chemistry, vol. 286, no. 24, pp. 21742-21754, 2011. 


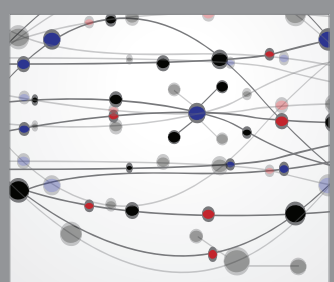

The Scientific World Journal
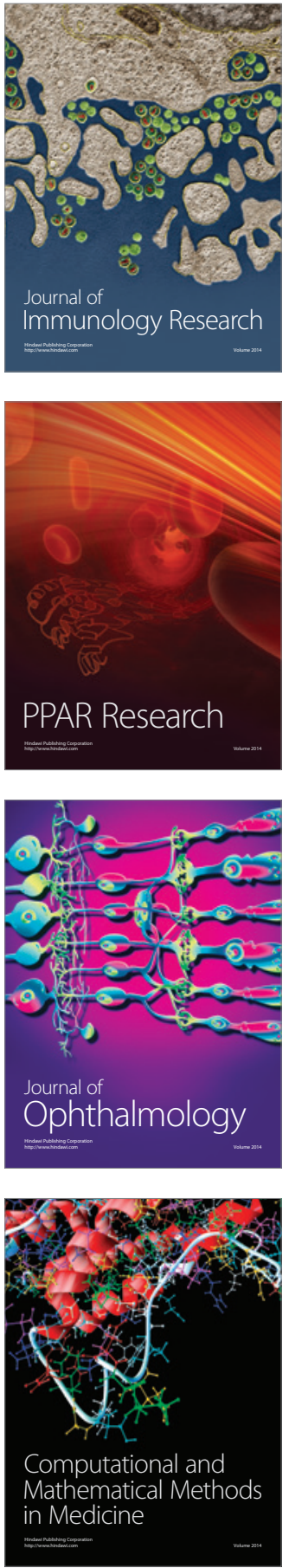

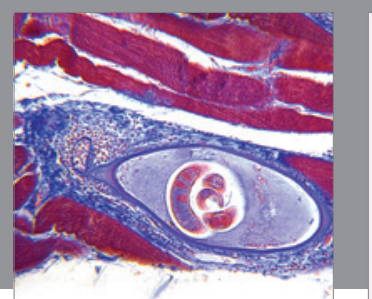

Gastroenterology Research and Practice

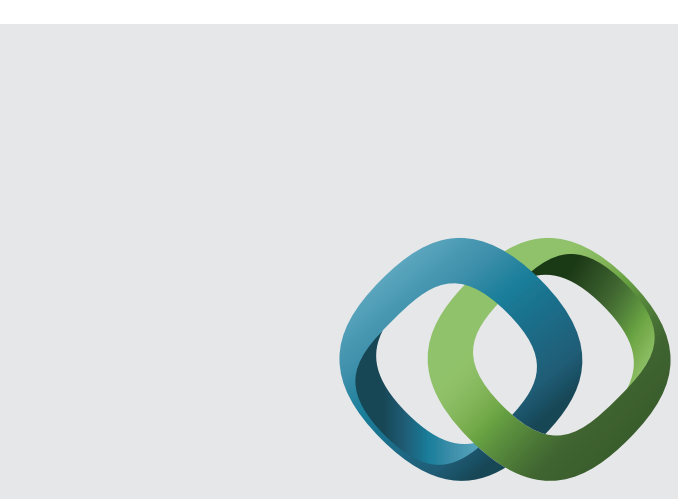

\section{Hindawi}

Submit your manuscripts at

http://www.hindawi.com
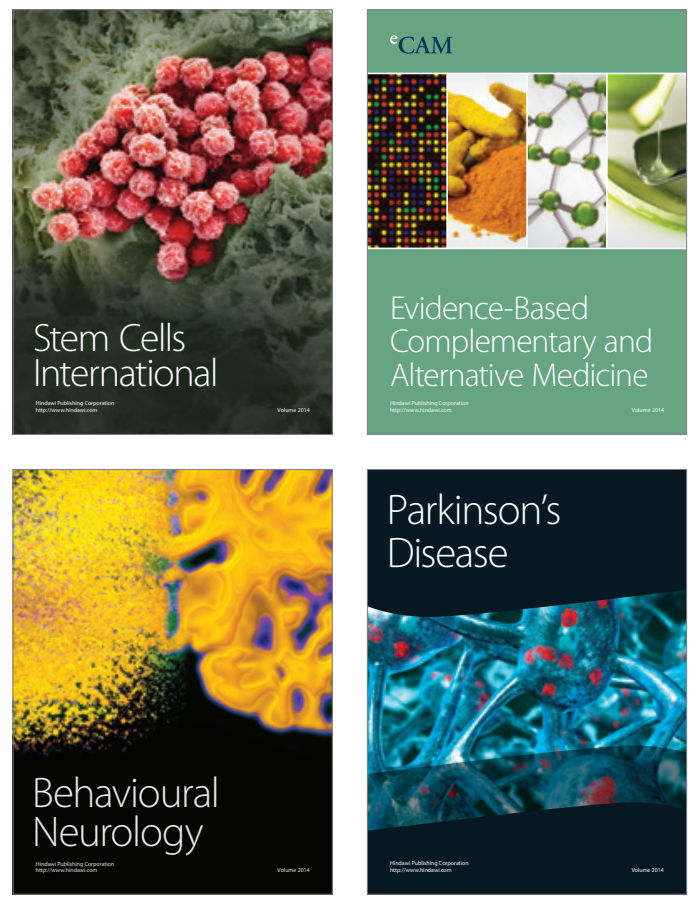
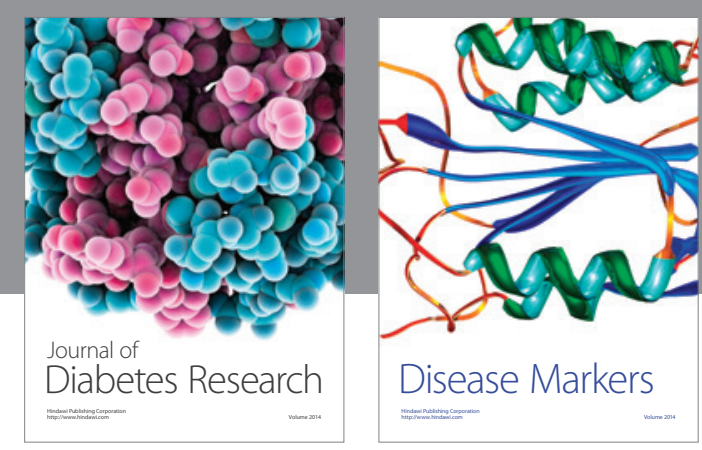

Disease Markers
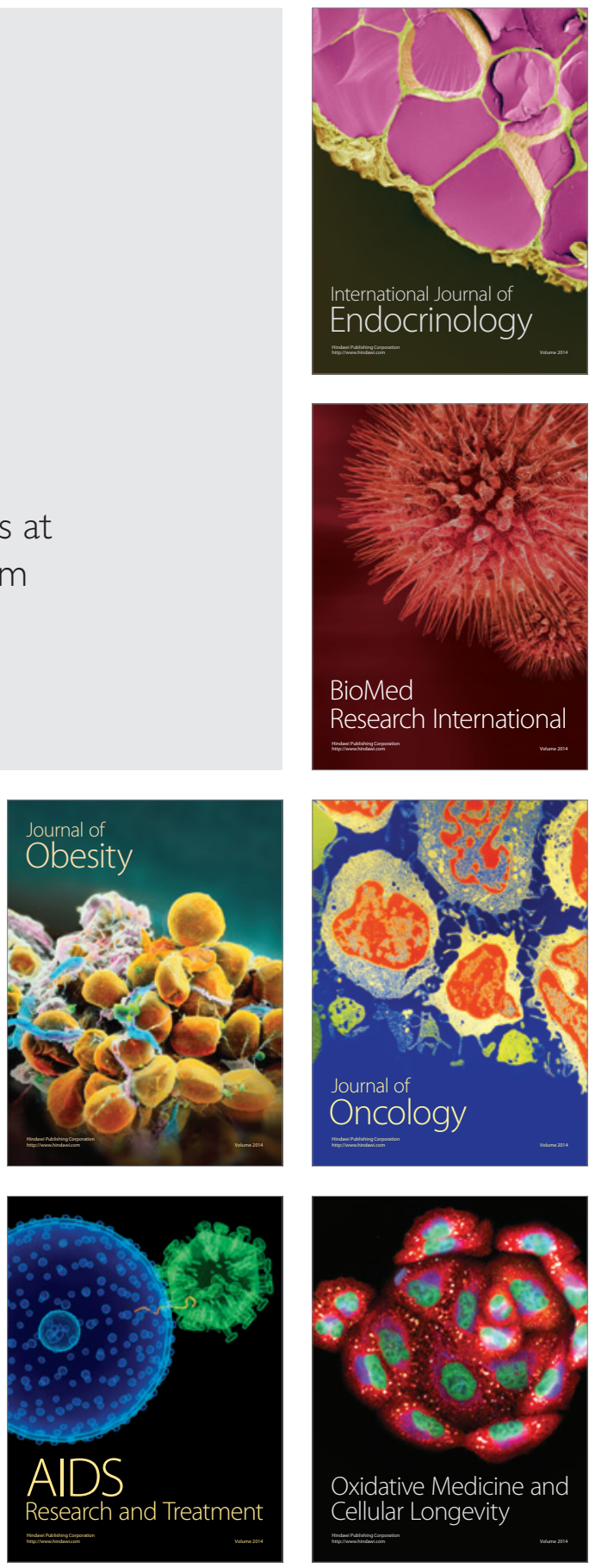